\title{
Analytical Modeling of LTE-based Network Capacity for Public Safety Communications
}

\author{
Kehinde O Olasupo ${ }^{*}$, Ivica Kostanic, Tajudeen O Olasupo
}

Electrical and Computer Engineering, Florida Institute of Technology, 150 W University Blvd, Melbourne, FL 32901, United States

Copyright $(2016$ by authors, all rights reserved. Authors agree that this article remains permanently open access under the terms of the Creative Commons Attribution License 4.0 International License

\begin{abstract}
In this paper, an analytical approach for estimating the network capacity for voice communications on LTE-based public safety networks is presented. The network is modeled using Markov birth and death process. In order to provide the required Grade of Service for LTE-based public safety users, the minimum number of channels required for different levels of users' priority is calculated. The results for some performance metrics such as throughput, delay probability, and channel utilization are obtained. The results obtained from the simulation is compared with the existing network model such as Erlang C. Results show that the existing network model show similarities and differences in predicting the channel estimation of the deployed LTE networks for public safety communications. The results can help the network designer in the proper dimensioning of LTE-based public safety network.
\end{abstract}

Keywords Public Safety, LTE, Delay Probability, Throughput, Grade of Service

\section{Introduction}

The public safety agencies such as the fire brigade, police, and emergency medical services (EMS) have over the years faced an increasing pressure on their safety operations due to growing natural and man-made disasters all over the world. The existing Private/Professional Mobile Radio (PMR) devices used by the agencies for public safety wireless communication systems are based on narrowband and wideband technology. These devices are frequently not enough to meet users' needs in many critical situations. The public safety agencies have acknowledged that in order to support full service capability and to improve operational efficiency during their operations, there will be need for broadband systems. The broadband systems support high data demanding services. Long Term Evolution (LTE) technology for mobile broadband public safety communications has been accepted as the technology suitable for future public safety communications. Public safety communication systems must be resilient and highly available, reliable, secure, and should be able to support point-to-multipoint communications. In public safety operations, the users in the field depend on effective communications for their safety and effectiveness. Both the transmitting and the receiving ends of the voice link should be able to clearly understand each other because minor errors in a voice call at critical times can be costly. Public safety users can be categorized as those whose daily operations are to ensure safety and welfare of people around the world. These include police, fire fighters, and emergency medical services [1]. The public safety agencies are also referred to as the 'First Responders'.

During response in the public safety operations today, the first responders utilize PMR devices such as Terrestrial Trunked Radio (TETRA), TETRA for Police (TETRAPOL), and Associated Public Safety Communication Officials (APCO) Project 25 for public safety voice communications. These systems are narrowband and initially designed to support voice and low bit data rate services. PMR networks are designed to meet emergency responders' unique public safety requirements and provide guaranteed priority access to responders. In addition to PMR, some emergency responders are using mobile data services and applications provided by commercial LTE carriers to share information and supplement their mission critical voice capabilities. [2].

In order to meet the needs of public safety communication users, some of the unique features of PMR (TETRA) standard are [3]:

- Group calls (wide area fast call set-up)

- Direct Mode Operation (DMO) allows communications between radio terminals without the intervention of network infrastructure.

- High level voice encryption to meet the security needs of public safety agencies

- Priority call ( an emergency call facility that gets through even if the system is busy)

There is a clear difference between PMR networks and commercial cellular networks (LTE). The operational needs of the users for the two networks are different. PMR systems 
make use of 'Push-to Talk' (PTT) radio systems. It also possess distinct security property such as end to end encryption, field control by a dispatcher, as well as trunking (switching) capability that make them different from cellular systems [4]. Also, there are some important TETRA requirements such as DMO, group calls, priority calls etc. that cannot be provided by the commercial mobile cellular networks. TETRA networks are designed to support data rates comparable to the second generation $(2 \mathrm{G})$ commercial cellular networks such as Global System for Mobile Communication (GSM) and General Packet Radio Service (GPRS). When disasters and emergencies occur, response organizations and government public safety agencies need prompt broadband communications support to deliver real-time updates of events, start relief operations, and provide continuing assistance in the affected regions. The introduction of data services in supplement to voice by the public safety agencies place is higher demand on the network from the capacity stand point. Hence, there is a need for broadband systems that support high data demanding services. There are various suggested options to handle increase in the traffic volume. Presently, Long Term Evolution (LTE) and LTE-Advanced; a generation of the mobile broadband technology after $3 \mathrm{G}$ technologies with much higher data rates of $100 \mathrm{Mbit} / \mathrm{s}$ (Release 8 and 9) and $1 \mathrm{Gbits} / \mathrm{s}$ (Releases 10 - 13) standards for mobile broadband is being recognized as the technology of choice for the future PPDR communications [5]. Also, technical work is currently being carried out within the 3rd Generation Partnership Project (3GPP) - the organization in charge of LTE standardization - to further improve the capabilities and features of LTE standard which will make it suitable for the requirements of PPDR and other professional users [6]

LTE, a standard for mobile phones and data terminals is based on the GSM and Universal Mobile Telecommunications Systems (UMTS) network technologies. It is an Internet Protocol (IP) - based systems designed to cope with the challenges of the growing broadband mobile market. LTE is continuously evolving to meet the needs of industry demands through 3GPP standardization activities. This permits the public safety communication personnel to take the advantage of LTE as mobile broadband networks for real - time data sharing, high quality video streaming, and quick response during emergencies and day-to-day activities. Hence, the situation awareness will be improved and safety of first responders will be better. Also, LTE- based public safety networks will allow use of many multimedia services such as text, voice, video, file/picture sharing etc. with quality, resilience and reliability [7].

Presently, there is limited research on traffic modeling for accurate capacity planning for LTE-based public safety networks either by simulation or measurement. This research thereby aims to develop an analytical traffic model for an optimal voice and data communications on LTE-based public safety networks. This will be in terms of number of channels, delay probability, overhead, and number of users in the network whereby guaranteeing the service level requirement (SLR) for the network.

This study does not consider the impact of higher layer (such as Network or Transport) on network capacity or QoS. It focuses on the radio interface. This is the PHY/MAC layer where the amount of information (throughput $=$ voice + data) from first responders that can access the communication channel per time without bringing down the network. It is a point to point communication consideration from the responder hand device to the nearest LTE-based public safety communication station.

The next section explains the related work and the theory behind the wireless broadband mobile technology. In section III, the options for deployment of mobile broadband for public safety are presented. Traffic modeling and network deployment optimization process are shown. Section IV explains the result. Finally, section V includes some conclusions.

\section{Related Work and Background}

\subsection{Literature Review}

There is no sufficient work in term of simulation or practical demonstration of deployment of LTE- based public safety network in literature. There are couple of white papers and technical reports that present various deployment options [8]. The process of deploying mobile broadband for public safety started in USA. The National Public Safety Telecommunications Council (NPSTC) and other organizations acknowledge the need for next generation public safety networks with broadband capabilities. LTE was chosen in 2009 for these public safety networks. The US government assigned band 14 at $700 \mathrm{MHz}$ of the spectrum to public safety communications. In 2012, the spectrum band is extended from $758-768 \mathrm{MHz}$ and $788-798 \mathrm{MHz}$ for LTE based public safety networks and all the responsibilities assigned to the First Responders Network Authority (FirstNet) for effective coordination [9, 10]. Currently, many other government organizations are proceeding to make use of LTE networks for public safety communications and services.

There have been many reports on the possibility of deployment of LTE networks for public safety. In [8], the white paper presents four options for the implementation of public safety mobile broadband capability. Also, it considers how the combinations of these options may provide a comprehensive capability. The options include: taking service from an existing commercial mobile network operator (MNO), operating as a mobile virtual network operator, taking service from a commercially owned dedicated network, and building, own and operate a dedicated network. The white paper also suggests the combination of these options. In the same vein, various options for delivering new generation of public safety devices $[11,12,13,14]$ are discussed. One option is to make 
use of upgraded commercial networks-LTE. The network deployment would be modified to meet the specific operational requirements of the public safety sector. Another option is to build a new generation of dedicated mobile broadband networks which would be used exclusively for public safety.

There are several studies on the traffic analysis to determine the performance metrics of trunked dispatch PMR systems. Hoang et al. $[15,16]$ present a system that models a central queue preceded by a group of queues. A decomposition method is used to solve the problems of both system and group delay. Likewise, Cackov et al. [17, 18] carry out extensive studies on the pattern and nature of traffic. They perform simulations and conclude that inter-arrival call is best modeled by an exponential distribution and call duration by a lognormal distribution. The technical case study in [19] discusses a simulation model on a multi-server queue with "First -In - First - Out" (FIFO) arrangement. The simulation is done with two scenarios: one is to model call inter-arrival with a gamma distribution and call duration by a lognormal distribution; and the second scenario models empirical data with an exponential distribution. They discover that both scenarios are almost the same in performance and close to the practical performance observed on the system. Finally, they use exponential distribution for both call inter arrivals and call duration, and use Erlang $\mathrm{C}$ for performance analysis of the central queue.

There are insufficient studies in the area of traffic modeling of PMR systems over LTE networks. Lin et al. [20] propose a model of Push-to-Talk over LTE system architecture. They use extended Erlang B formula to analyze the blocking probability of calls. They do not consider what traffic analysis would be when the calls are put in a queue instead of being blocked, only for the users to try again after each call failure.

Ali et al. [21] investigate the Session Initiation Protocol (SIP) signaling performance over LTE- based mission-critical communications and services. They define the performance metrics for overall system using discrete event simulator. However, there is no traffic modeling done for the estimation of calls delay. Though, the estimation of network performances of TETRA-based public safety network is done in [22] by simulating the traffic using a computer tool; there is still need for further research in estimating the traffic of LTE-based public safety networks. With insufficient research in this area, there is need for further research in estimating the traffic of LTE-based network for public safety.

\subsection{LTE for Public Safety Communications}

LTE, a technology after $3 \mathrm{G}$ was developed by $3 \mathrm{GPP}$ to keep mobile communication systems competitive for longer years in to the future with high data rates and low latencies that users would require. One of the important factors that make LTE to be desired for mission critical communications is the economies of scale when compared with existing
PMRs like TETRA. There will be reduction in both the Capital Expenditure (CAPEX) - infrastructure costs and the Operational Expenditure (OPEX) - operational costs. In addition to this, LTE has the ability to provide efficient high speed, low latency, low set- up time, and high- security data connectivity. All these factors are needed to establish public safety multimedia communications. Another factor in adopting LTE is the availability of radio equipment for different deployment scenarios. LTE base stations are available to cover different cell sizes such as macro, micro, pico and femto. With diverse cell size, public safety networks can be easily set up in the rural areas where there is no coverage of a macro cellular network.

LTE will continue to evolve further in both capabilities and performance. The further improvements are described in Releases by 3GPP, with new features for reliability resilience, scalability and management. LTE-Advanced is included in Releases 10 and 11 with speeds of $1 \mathrm{Gbit} / \mathrm{s}$. Additional enhancements are described in releases 12-14, all paving the way for $5 \mathrm{G}$ that would give a higher data throughput. Some basic 3GPP features for public safety have been incorporated in Releases 8 -11 of LTE standards; such as prioritization and emergency calls. New solutions for public safety are included in Releases 12 which include proximity services (Device-to-Device, or D2D) which facilitate the discovery and communications between nearby radio users without the network infrastructure. Also, group communication that allows one-to-many voice calls as well as dispatcher communications is made available in Release 12 [23, 24]. Presently, Enhanced proximity services and Mission critical push-to-talk is available in Release 13 [25].

The evolution stages in the existing PMR-TETRA and the commercial cellular networks (CCN) are illustrated in Fig 1. The figure also indicates an increasing data rate for each stage of evolution. Both $3.9 \mathrm{G}$ and $4 \mathrm{G}$ stand for 'Broadband' as the overall name in the wireless communications systems.

\begin{tabular}{|c|c|c|c|c|c|c|}
\hline $\begin{array}{l}3 \mathrm{GPP} \\
\mathrm{CCN}\end{array}$ & $2 \mathrm{G}$ & $2.5 \mathrm{G}$ & $2.75 \mathrm{G}$ & $3 \mathrm{G}$ & $3.8 \mathrm{G}$ & $3.9 \mathrm{G}$ \\
\hline & GSM & GPRS & EDGE & UMTS & $\mathrm{HSPA}^{+}$ & LTE LTE-A \\
\hline ETSI & TE TF & A1 & TETRA? & & & TETRA 3 \\
\hline TETRA & $\begin{array}{r}\mathrm{V}+\mathrm{D}, \\
1 \mathrm{kbps}\end{array}$ & DMO & $\begin{array}{l}\text { TEDS } \\
\text { Data }\end{array}$ & Rate & & $\begin{array}{r}\text { TE TRA-L TE } \\
1 \mathrm{Gbps}\end{array}$ \\
\hline
\end{tabular}

Figure 1. Evolution in TETRA and Commercial Cellular Networks $(\mathrm{CCN})$

\section{Proposed Deployment Options}

\subsection{Deployment and System Setup}

Public Safety networks have been designed as standalone networks that are usually deployed by the government to assure privacy and security of the networks. Deployment of networks in this way is often costly as high quality, high 
reliability and security are required. One deployment option for LTE for public safety is for the government to build, own and operate dedicated network - a standalone private LTE for public safety. Consequently, the government has the full control of the network $[8,14]$.

Second option is the development of Random Access Network (RAN) sharing mechanisms for public safety. If there is an agreement between the existing public safety network operators and commercial mobile operators to share radio base stations and also the sites where the base stations are physically deployed, the public safety agencies could benefit from the coverage and capacity of LTE networks [14].

Another deployment scenario is integrating public safety network over mobile broadband. The easiest and probably the cheapest option is the public safety network taking service from an existing commercial MNO [8]. The deployment option of integrating public safety over mobile broadband is shown in Fig. 2. This deployment option is assumed in the proposed analytical simulation. The Application Server (AS) of the existing public safety network is connected to the Packet data network gateway (PGW) of LTE. Currently, LTE networks already exist in many countries and public safety users can make use of this opportunity. The option can also be a cost effective solution in the sense that there will be low CAPEX on infrastructure. Also, operational expenditures will be low since the network would be supporting commercial users as well as public safety users.

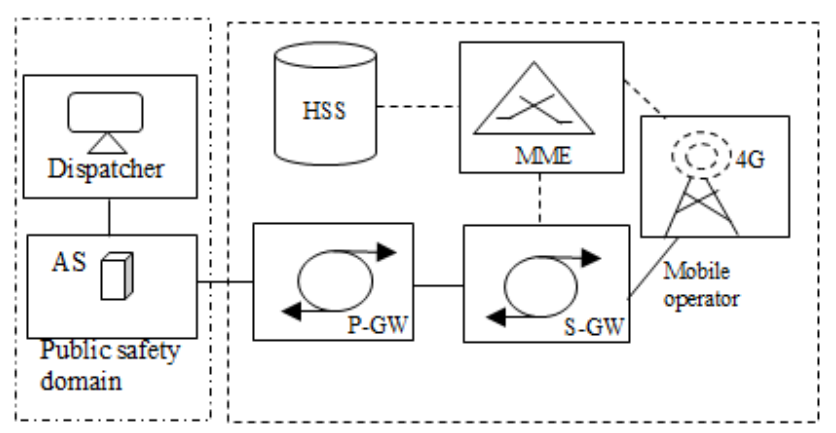

Figure 2. Deployment of Public safety over mobile broadband

\subsection{Traffic Modeling and Network Deployment Process}

\subsubsection{Traffic Modeling}

Traffic can be seen as the driving force behind all telecommunications activities. The probability models for the traffic flow are necessary for predicting the system performance metrics to an acceptable degree of accuracy. Also, traffic modeling is needed to determine the operational status of the networks, evaluate their performance, and to easily detect possible network congestion.

Therefore, the basic aim of traffic modeling of public safety communications over LTE networks is the prediction of network performance. Using the queuing theory model, voice traffic randomly arrives at the channel with service requirements which may be random in duration. The theory tries to find probabilistic descriptions of such quantities as the sizes of queue, the delay experienced by an arrival, and the availability of a channel. The queue follows Markov birth-death model and a FIFO discipline. The queue system considered is a multi-server with $N_{k}$, the number of channels, mean rate of call arrival, $\lambda$, and the mean call duration $\frac{1}{\mu}$. Performance metrics are based on the statistics obtained for the central queue. These include delay probability (i.e. probability of waiting in the queue for channel access), $\mathrm{P}_{\mathrm{d}}$, and average waiting time (or queuing delay) for those calls that have to wait in the central queue, $\mathrm{T}_{\mathrm{d}}$. These performance metrics are used to define the Grade of Service (GoS) for LTE-based public safety networks.

\subsubsection{Modeling Traffic Volume}

The process of modeling involves voice traffic arriving a server or channel. Probability theory is used to determine the channel needed and the associated delay time. Call or traffic categories can be mobile to mobile (M-M), mobile to external fixed terminals (M-F), and others.

For a single cell site consideration, the probability that the call traffic will be delayed more than a particular time $t$ is

$$
P d_{N_{k(>t)}}=P d_{N_{k(>0)}} \times e^{-\frac{\left(N_{k}-N_{C}\right) \times t}{T_{h}}} \text {. }
$$

where $P d_{N_{k(>0)}}$ is the probability that a call will be delayed, $N_{k}$ is the number of trunks available for offered traffic, $N_{c}$ is the volume of traffic in Erlang, $T_{h}$ is the call duration or holding time in (s).

Assume $P d_{N_{k(>t)}}$ is similar for $k$ sites, then, $P d_{N_{k(>t) t o t a l}}$, the probability that all $k$ sites are involved in call after a certain waiting time is [4]

$$
P d_{N_{k(>t) t o t a l}}=1-\left(1-P d_{N_{k(>t)}}\right)^{k}
$$

where $k$ is the total numbers of cell sites involved in the call. During major incidence and accidents, the public safety agencies are always in a location which can be served by one or two cell sites. Therefore, (1) will be used in the traffic modeling simulations.

Similarly, the total traffic offered will be:

$$
N_{c}=u \times \lambda \times T_{h} \times m .
$$

where $u$ is the total numbers of users per cell site involved in the call. $\lambda$ is the busy hour call attempts per subscriber in (call/s), $m$ is of cells involved in the group call.

\subsubsection{Modeling Number of Traffic Channels}

The traffic modeling process assumes infinites population source where the ratio of user to available channel is 20:1. The incoming call arrival rate follows Poisson distribution. It also assumes that the system is in equilibrium with equal traffic density per source. The incoming traffic has exponentially calls holding time. Blocked requests are delayed or queued, and calls are served in order of arrival with exception of pre-emptive priority calls and other priority call levels. Call arrival follows $\mathrm{M} / \mathrm{M} / \mathrm{C}$ queueing 
system as shown in Figs.3. M/M/C denotes a system with Poisson call arrivals, exponentially distributed service times and $\mathrm{C}$ identical servers/channels in parallel. The system does not reject any call request. Markov process of Birth- death rate is used to model delay probability for when the system is in equilibrium as seen in Fig. 4.The simulated data from scenario 8 of ETSI technical paper [26] is used in the analysis of traffic modeling for LTE-based public safety network.

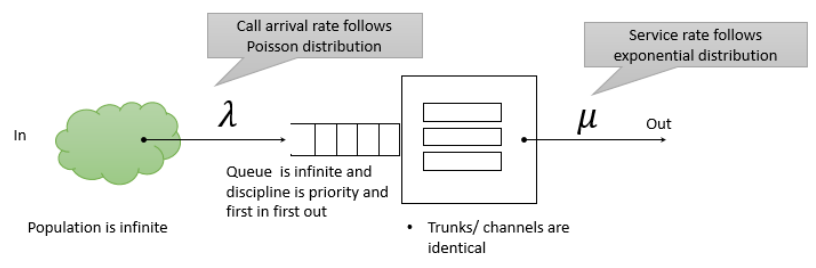

Figure 3. $M / M / C$ queueing system

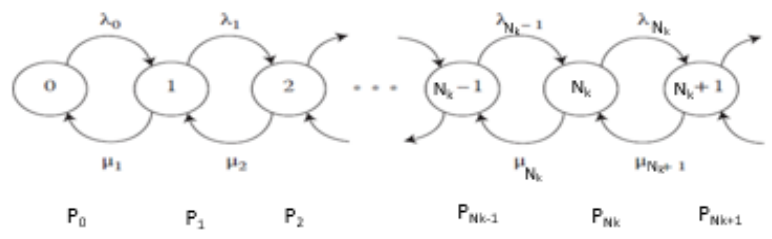

Figure 4. Markov process of birth- death rate

Using the Markov process of birth- death rate model, from Figs. 3 and 4, traffic flow rate in to state $N_{k}$ is equal to traffic flow rate out of state $N_{k}$ for equilibrium state. The probability $P_{N_{k}}$ is the probability of being in the state $N_{k}$.

For idle trunk state or state 0 ,

$$
\mu_{1} P_{1}=\lambda_{0} P_{0}
$$

For one trunk available or state 1 ,

$$
\lambda_{0} P_{0}+\mu_{2} P_{2}=\left(\lambda_{1}+\mu_{1}\right) P_{1}
$$

For $N_{k}$ numbers of trunk available or state $N_{k}$,

$$
\lambda_{N_{k}-1} P_{N_{k}-1}+\mu_{N_{k}+1} P_{N_{k}+1}=\left(\lambda_{N_{k}}+\mu_{N_{k}}\right) P_{1}
$$

Accordingly, the sum of all probability of possible system population add to $100 \%$ that is

$$
\sum_{N_{k}=0}^{\infty} P_{N_{k}}=1
$$

Similarly, the probability that an incoming request find at least one trunk is

$$
P_{N_{k(>0)}}=\sum_{N_{k}=0}^{N_{k}-1} P_{N_{k}}
$$

Also for the probability of delay $P d_{N_{k}(>0)}$ will be:

$$
P d_{N_{k(>0)}}=1-\sum_{N_{k}=0}^{N_{k}-1} P_{N_{k}}
$$

If $P d_{N_{k(>0)}}$ is known or given as part of Service Level Agreement (SLA), therefore the probability that call will be answered or served less than a particular time $t$, and a call holding time in (s), will be

$$
P d_{N_{k(<t)}}=1-P d_{N_{k(>0)}} e^{-\frac{\left(N_{k}-N_{C}\right) \times t}{T_{h}}}
$$

Or for an exceeding time,

$$
P d_{N_{k(>t)}}=P d_{N_{k(>0)}} e^{-\frac{\left(N_{k}-N_{c}\right) \times t}{T_{h}}}
$$

Furthermore, the SLA for PMR-TETRA to meet its design is that probability of call delay exceeding, a time of $5 \mathrm{~s}$ is $5 \%$ [27] that is, $P d_{N_{k(>5)}}=5 \%$.

Substituting $P d_{N_{k(>5)}}=5 \%$ into (9), it becomes,

$$
\frac{5}{100}=P d_{N_{k(>0)}} e^{-\frac{\left(N_{k}-N_{C}\right) \times 5}{T_{h}}}
$$

Apply law of natural logarithm to (12) and simplify, that is,

$$
\text { given } x=e^{-y} \text {, then } y=-\ln (x)
$$

Equation (12) becomes:

$$
N_{k}=N_{c}-\left[\frac{T_{h}}{5} \times \ln \left(\frac{0.05}{P d_{N_{k}(>0)}}\right)\right]
$$

where $N_{k}$ is the trunk available for the offered traffic, $N_{c}$

For a given $P d_{N_{k(>0)}}$ such as $1 \%$ or $10 \%$, and total offered call traffic at a particular call holding time, the required traffic channel for the service can be obtained using (14).

Similarly, the delay, $T_{d}$ for traffic will be:

$$
T_{d}=\frac{P d_{N_{k}(>0)} \times T_{h}}{N_{k}\left(1-\rho_{N_{k}}\right)}
$$

where $\rho_{N_{k}}=\frac{N_{C}}{N_{k}}$ is the trunk or channel utilization

\subsubsection{Modeling Broadband Capacity for Public Safety Service (PSS) Traffic Volume and Real Time Operation using LTE Service}

Considering the option 3 deployment strategy discussed in section 3.1, the bandwidth capacity per cell site will be given as (Fig. 2) [4]:

$$
B=C \times B_{w} \times R
$$

where $C$ is the carrier capacity per cell site, $B_{w}$ is trunk bandwidth, and $R$ is the reuse factor.

Therefore, from Fig.5, the bandwidth capacity for TETRA and LTE respectively will be:

$$
\begin{gathered}
B_{1}=C_{1} \times B_{w 1} \times R_{1} \\
B_{2}=C_{2} \times B_{w 2} \times R_{2}
\end{gathered}
$$

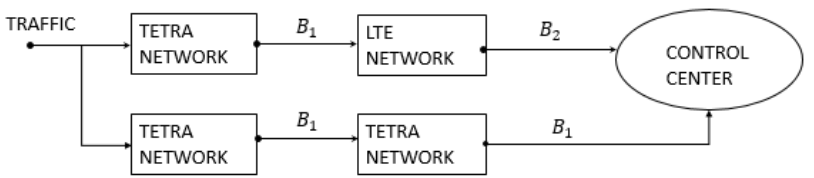

(a) 


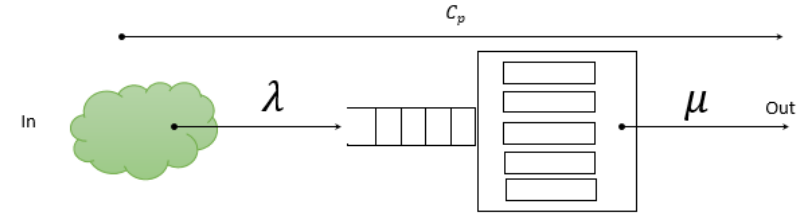

(b)

Figure 5. (a) Bandwidth capacity deployment plan for TETRA and LTE (b) Queue system for bandwidth capacity in LTE- based public safety network

The proposed LTE trunk bandwidth is $10 \mathrm{MHz}$ and the existing value for PMR-TETRA is $25 \mathrm{KHz}$, the reuse factor for LTE is higher than that of TETRA, [4], [5], [27], assuming it is double, and $C_{1}=C_{2}$, and divide (18) by (17), therefore,

$$
\gamma=\frac{B_{2}}{B_{1}}=\frac{C_{1} \times 10 \times 10^{6} \times 2 R_{1}}{C_{1} \times 25 \times 10^{3} \times R_{1}}=800
$$

where $\gamma$ is a bandwidth constant.

Similarly the service throughput $T_{p}$ (in $S^{-1}$ ) can be given as

$$
T_{p}=\mu-\lambda
$$

Provided other factors remain constant, (16) becomes

$$
C_{p}=\gamma T_{p}
$$

where $C_{p}$ is the traffic capacity or broadband service

Presently, Commercial LTE is capable of $100 \mathrm{Mbps}$ in the downlink and $50 \mathrm{Mbps}$ in the uplink and most of our videos are downloads. But, the case is different for LTE - based Public Safety Communication (PSC) network, the uplink (as shown in Figure 6) is of paramount importance because the first responders transmit high information on upload link than the download. The uplink uses single carrier frequency division multiple access (SC-FDMA).

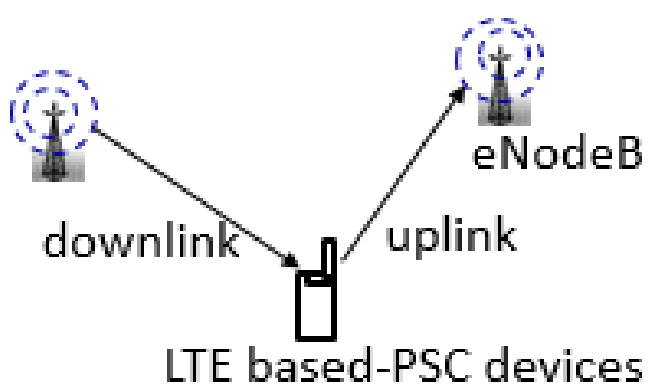

Figure 6. LTE- based Public safety communications (LTE- based PSC) radio air interface.

There are three physical layer channels defined for the uplink in LTE. These are Physical Uplink Shared Channel (PUSCH), Physical Uplink Control Channel (PUCCH), and Physical Random Access Channel (PRACH). PUSCH carries user data and $\mathrm{PUCCH}$ is used for Control signaling. PUCCH comprises the uplink data transmitted independently of traffic data. The PRACH carries the random access preamble that UE sends to access the network in non-synchronized mode. It is used to allow the UE to synchronize timing with the eNodeB. It consists of six Resource Block [28].

Consequently, to calculate the estimate throughput of the proposed LTE based Public safety communications,

Firstly, the study determines the number of Resource Elements $(R E)$ per $1 \mathrm{msec}$ subframe given as:

$$
\begin{gathered}
R E=\text { Subcarriers } \times S C-F D M A \text { sysmbol } \times \\
\times \text { Resource block } \times \text { Slots }
\end{gathered}
$$

Then, the data rate (DR) when assuming 64QAM with Single Input Single Output (SISO) antenna configuration and no coding is:

$$
D R=\text { Number of bits per 64QAM symbol } \times R E
$$

The overhead related to PUCCH, PRACH, pilot, random access, control plane, guard band overhead and other channels is approximately $71.55 \%$, [6]. Therefore, the peak data rate or throughput is $71.55 \%$ of $D R$.

\section{Simulation Analysis}

\subsection{Analysis to Test Proposed Traffic Simulation Modeling}

\subsubsection{Traffic Volume and Required Trunks}

The simulated data from scenario 8 of ETSI technical paper [26] is used in the analysis of traffic modeling for LTE-based public safety network. The mobile to mobile (M-M) call rate for the simulation in Fig. 5a is given as 0.324 call/hour and the call holding time is 20 s. Using (3), the traffic volumes for different user are given in Table 1 . This study uses different priority levels with the associated probability of delay or Grade of Service (GoS) as shown in Table 2 to test the performance of the simulation model.

Similarly, using (14), numbers of trunks that will be required for LTE-based public safety network to be able to carry traffic volumes in Table 1 and delay requirements in Table 2 are obtained as shown in Table 3 . The plot of number of trunks obtained versus offered traffic at different grade of service is shown in Fig. 7. The plots show that higher number of trunks need to be available as traffic volume increases and at least for $99 \%$ of the time at least a trunk must be available for preemptive calls.

Table 1. Traffic Volumes for different Public Safety Network users

\begin{tabular}{|c|c|}
\hline No of users & Traffic volume (Erlang) \\
\hline 100 & 0.180 \\
\hline 250 & 0.450 \\
\hline 300 & 0.540 \\
\hline 500 & 0.900 \\
\hline 750 & 1.350 \\
\hline 1000 & 1.800 \\
\hline
\end{tabular}

Table 2. Different Priority levels and the associated Grade of Service (GoS) 


\begin{tabular}{|c|c|}
\hline Priority delay requirements & GoS Value (\%) \\
\hline Pre-emptive priority & 1 \\
\hline Priority \#1 & 10 \\
\hline Priority \#2 & 25 \\
\hline Priority \#3 & 50 \\
\hline Priority \#4 & 71 \\
\hline Priority \#5 & 89 \\
\hline
\end{tabular}

Table 3. Simulated Number of Traffic Trunks for LTE-based Public Safety Network for given SLA

\begin{tabular}{|l|l|l|l|l|l|l|}
\hline & \multicolumn{7}{|c|}{ Number of trunk } \\
\hline $\begin{array}{l}\text { Probability } \\
\text { of delay }\end{array}$ & $1 \%$ & $10 \%$ & $25 \%$ & $50 \%$ & $71 \%$ & $89 \%$ \\
\hline $\begin{array}{l}\text { Traffic } \\
\text { Erlang }\end{array}$ & \multicolumn{7}{|c|}{$\%$} \\
\hline 0.18 & 12 & 12 & 11 & 9 & 7 & 3 \\
\hline 0.45 & 12 & 12 & 11 & 10 & 7 & 4 \\
\hline 0.54 & 12 & 12 & 11 & 10 & 8 & 4 \\
\hline 0.90 & 13 & 12 & 12 & 10 & 8 & 4 \\
\hline 1.35 & 13 & 13 & 12 & 11 & 8 & 5 \\
\hline 1.80 & 14 & 13 & 13 & 11 & 9 & 5 \\
\hline
\end{tabular}

Table 4. Comparison between Simulation Model and Erlang C table for Number of Trunks

\begin{tabular}{|c|c|c|c|c|c|c|}
\hline Probability of delay & \multicolumn{7}{|c|}{$89 \%$} \\
\hline Traffic (Erlang) & 0.18 & 0.45 & 0.54 & 0.90 & 1.35 & 1.80 \\
\hline Simulation model & 3 & 4 & 4 & 4 & 5 & 5 \\
\hline Erlang C table & 2 & 2 & 2 & 3 & 4 & 4 \\
\hline
\end{tabular}

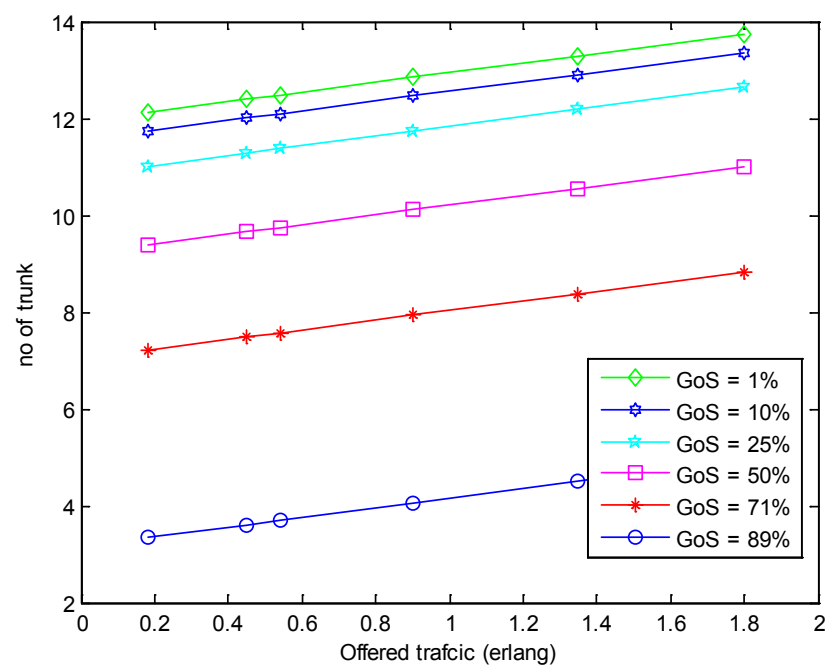

Figure 7. Number of trunk versus offered traffic at different grade of service

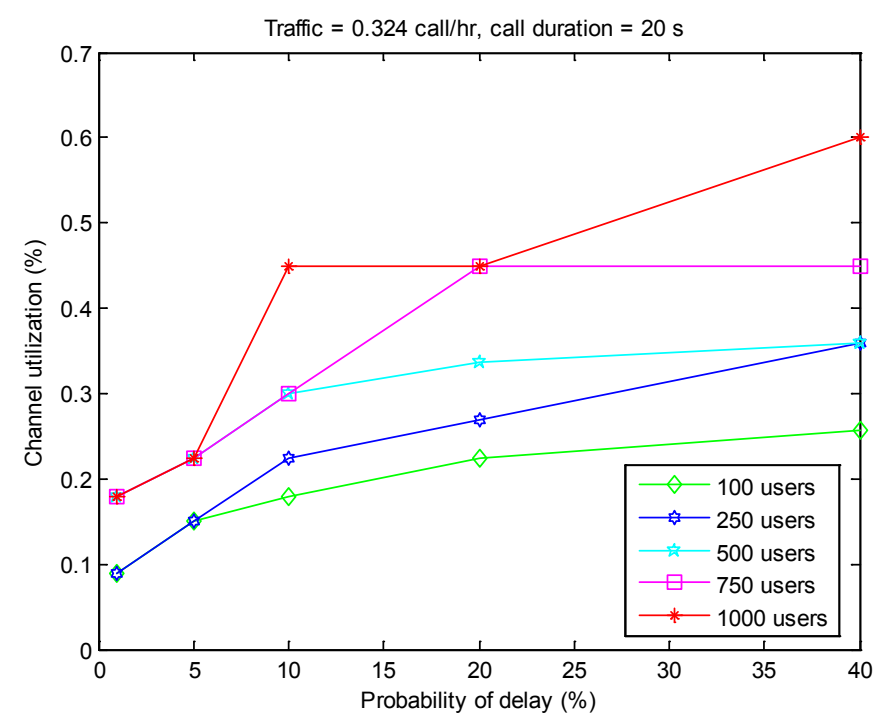

Figure 8. Channel utilization versus probability of delay

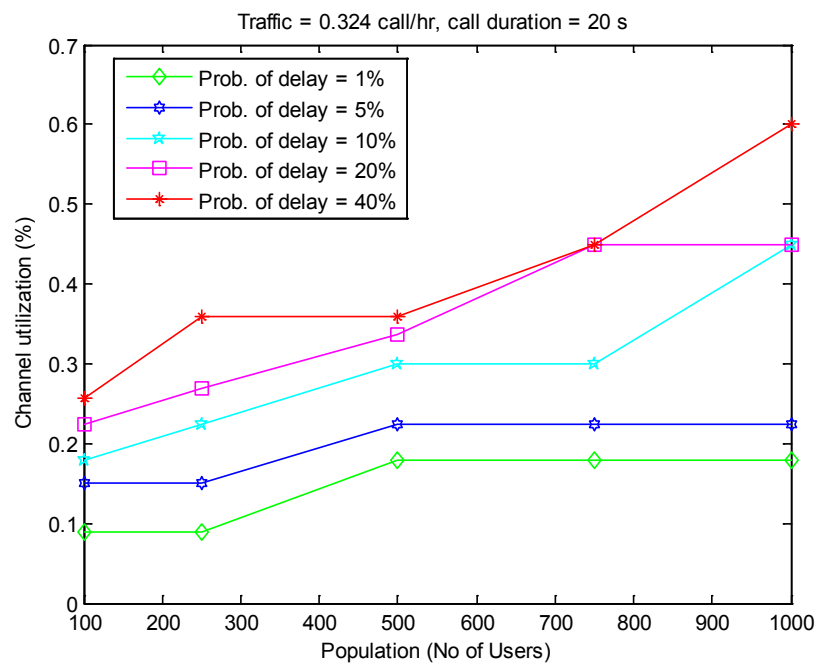

Figure 9. Channel utilization versus number of users

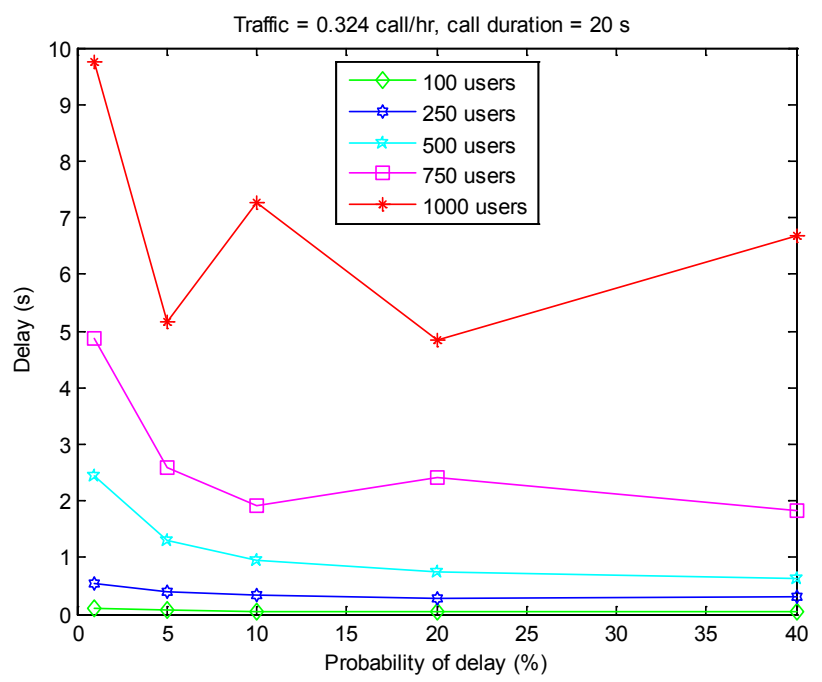

Figure 10. Waiting time versus probability of delay 


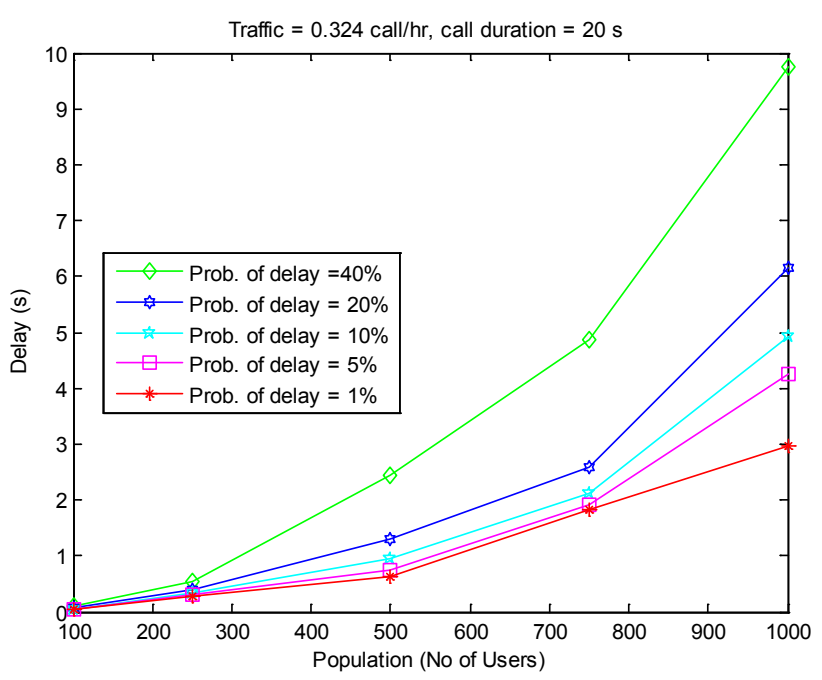

Figure 11. Waiting time versus number of users

The plots for channel utilization based on the traffic versus the probability of delay and number of users is shown in Figs. 8 and 9 respectively. The plots show that channel utilization is low for pre-emptive calls. The channel utilization plots give us an excellent view of performance of a channel. As the channel utilization statistic is low for pre-emptive calls, the network is doubtless in pretty good shape. Similarly, the plots for delay versus the probability of delay and number of users are shown in Figs. 10 and 11 respectively. The plots show that the waiting time increases tremendously as the number of users increase for given number of trunks. The longer this performance measure is, the worse would be the perception of the network from the public safety users' points of view.

\subsubsection{Calculating Time and Test of Broadband Capacity}

From the data sample obtained from the ETSI report, it can be shown that:

$$
\begin{gathered}
\mu=\frac{1}{T_{h}}=\frac{1}{20}=0.05 \mathrm{~s}^{-1} \\
\lambda=\frac{0.324 \mathrm{call}}{h r}=\frac{0.324}{3600}=9.05 \times 10^{-5} \mathrm{~s}^{-1}
\end{gathered}
$$

Using (19),

$$
\begin{gathered}
T_{p}=\mu-\lambda \\
T_{p}=0.05 s^{-1}-9.05 \times 10^{-5} s^{-1} \\
T_{p}=0.04991 s^{-1}
\end{gathered}
$$

Using (20)

For the link in Fig 5 (b),

Existing PMR-TETRA capacity, $C_{p}=\gamma T_{p}$

$$
C_{p}=0.04991
$$

Similarly, for LTE-based public safety network capacity,

$$
\begin{gathered}
C_{p}=\gamma T_{p} \\
800 \times 0.04991 \approx 40 s^{-1}
\end{gathered}
$$

Therefore, the capacity ratio of LTE-based public safety network to conventional PMR-TETRA is 40:1.

Also, the time required for traffic transmission for the existing PMR- TETRA is $\frac{1}{0.04991} \approx 20 s$ while the time required for traffic transmission in LTE-based public safety network is $\frac{1}{40} \approx 25 \mathrm{~ms}$.

From this analysis, it is shown that for a LTE-based public safety network, call or message transfer will take approximately $25 \mathrm{~ms}$.

Furthermore, in an accident scene where a user has incident user video of $768 \mathrm{kbps}$ peak rate and other data transfer of $512 \mathrm{kbps}$, the total date rate (peak traffic) for the uplink is $1300 \mathrm{kbps}$ [29]. This means the generated PSC traffic is $1300 \mathrm{kbps}$. Using this data, the LTE capacity can be obtained using equations 22 and 23. For $2 \times 10 \mathrm{MHz}$ bandwidth assigned for the public safety communications, there are 12 subcarriers, 7 SC-FDMA per symbol and 16 resources block for 2 slots [28, 29].

Therefore, the LTE capacity is obtained by calculating the peak data rate as illustrated below.

$$
R E=\frac{12 \times 7 \times 16 \times 2}{1 \times 10^{-3}}=2688000
$$

$$
\begin{gathered}
\text { Peak data rate }=0.7155 \times 6 \times 2688000 \\
=11.54 \text { Mbps }(28)
\end{gathered}
$$

Analytically, the throughput that can be obtained for $2 \mathrm{X}$ $10 \mathrm{MHz}$ LTE network is $11.54 \mathrm{Mbps}$ for SISO antenna configuration. Therefore, with PSC traffic of $1300 \mathrm{kbps}$, the LTE network is capable of carrying 8 times the traffic.

\subsubsection{Evaluation of Cost of Deployment of LTE Network for Public Safety Communication}

If the existing PMR-TETRA is integrated over commercial LTE network for public safety, this in turn will provide speed and broadband services for real time services with higher throughput. These benefits come with a cost. Such as, cost of migration and hardware/software upgrade and optimization, running and maintenance cost. Capital Expenditures (CAPEX) and Operational Expenditures (OPEX) are the total expenditures for the deployment of a wireless mobile network.

For a given SLA, let us assume there are two traffic plans or usage rates, that is:

Plan A: Priority or preemptive calls with $\$ 30$ per hour per traffic

Plan B: Other emergency calls with $\$ 20$ per hour per traffic

Lease/dedicated trunk with $\$ 400$ flat rate.

Using traffic volume of 1.35 Erlang from Table 1, the plot of cost per time for the plans is shown in Fig. 12, in order to optimize the cost of implementation of LTE-based public safety network, the cost (OPEX) should stay below the curves. 


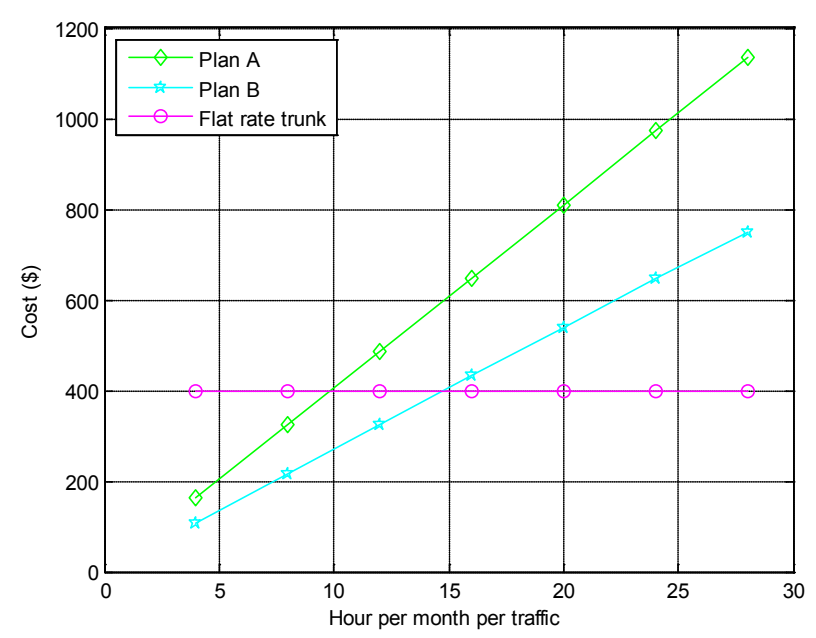

Figure 12. Plot for cost of deployment of LTE network for public safety

\subsection{Comparison of Analytical Traffic Simulation Model with Erlang C}

Table 3 shows the number of simulated trunks that will be required for LTE network for public safety. The simulation model to determine the number of required trunks for LTE-based public safety network is compared with the Erlang C table values. Analysis of Variance (ANOVA) is used to test the significance of the comparison. In the ANOVA analysis, the null hypothesis is that the mean values of number of trunks obtained by computer simulation model is the same as the mean values of number of trunks obtained by Erlang $\mathrm{C}$ table given a certain traffic and grade of service. The alternative hypothesis is that the mean value of number of trunks for computer simulation model and Erlang $\mathrm{C}$ table is different. Table 4 shows the number of simulated trunks and the ones obtained using Erlang $\mathrm{C}$ table. The result of ANOVA as shown in Fig. 13 shows that the p-values, 0.025 is less than the chosen significance level, 0.05 . Therefore this study rejects the null hypothesis. The Erlang C tables underestimate the amount of trunks required for TETRA network over LTE network. Similarly, Figs. 14 and 15 show the Tukey and the Hsu simultaneous data comparison respectively. They show that there is a difference in the number of required trunks for computer simulation model and Erlang $\mathrm{C}$ table. The Erlang $\mathrm{C}$ tables are not precise in the prediction and that they do not take into account the time requirement for a specific traffic at given grade of service.

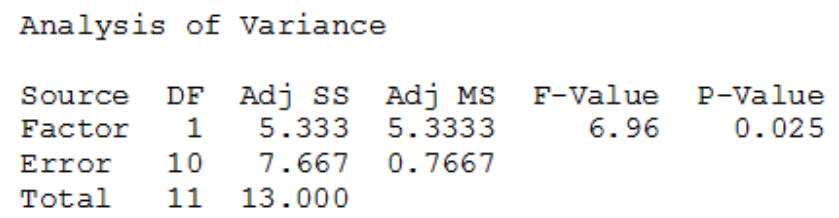

Figure 13. ANOVA result

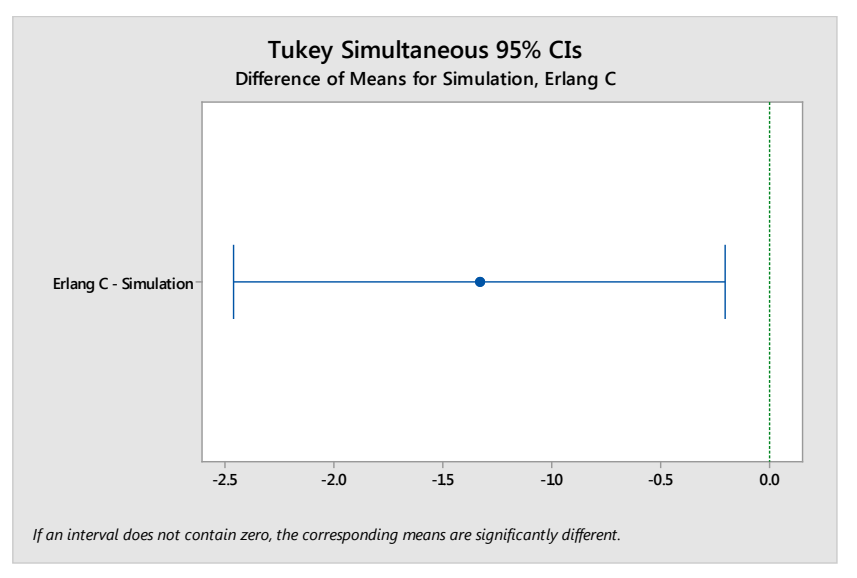

Figure 14. Plot for Tukey simultaneous data comparison

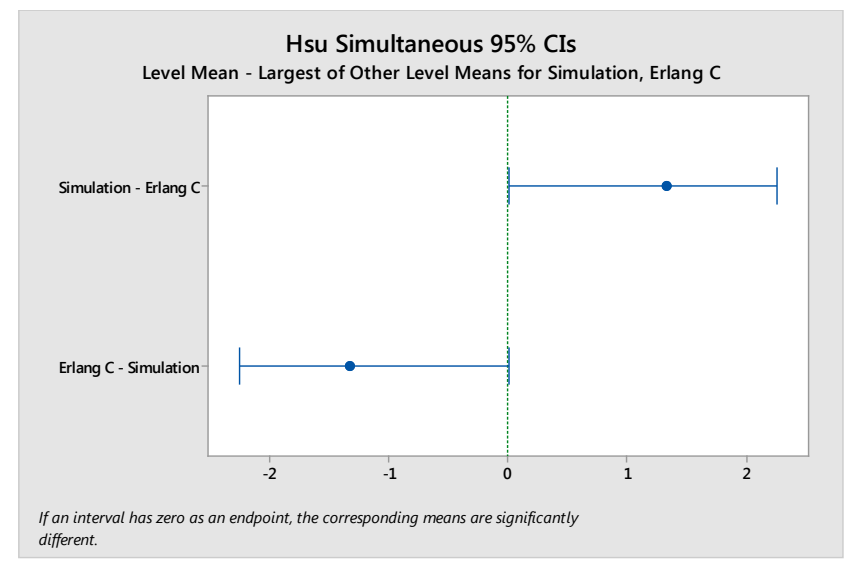

Figure 15. Plot the Hsu simultaneous data comparison

\section{Conclusions}

This study reports the traffic analytical modeling for proposed deployment of LTE network for public safety communications. The study demonstrates that deploying public safety networks over conventional commercial LTE networks will improve traffic capacity of such network and also make mission-critical services available on real time. It also shows the likely number of trunks that will be required form LTE network to meet the SLA for public safety network and also likely delays. Finally, the study presents the effect of optimizing network in term of cost as a result of deployment over LTE. To optimize the transition, the OPEX has to go below certain level. The future study will be to investigate the traffic characteristic and performance of other deployment options including DMO and group communications through simulation and measurement.

\section{REFERENCES}

[1] TCCA, "The Strategic Case for Mission Critical Mobile Broadband: A review of the future need of the users of critical communications," TETRA and Critical Communication Broadband Group, White Paper, 2013. 
[2] FirstNet, "First Responder Network Authority," 2012. [Online]. Available: http://www.firstnet.gov/network/lmr. [Accessed Monday August 2016].

[3] ETSI, "European Telecommunication Standard Institute," 1996. www.etsi.org/technologies-clusters/technologies/tetra. [Accessed 17 August 2016].

[4] P. Stavroulakis, Terrestrial Trunked Radio- TETRA: A global security tool, Springer, 2007.

[5] N. N. a. Solutions, "LTE Network for public safety services," Nokia Networks White Paper, 2014.

[6] 3GPP, "3rd Generation Partnership Project," 2008 -2016. [Online]. Available: www.3gpp.org. [Accessed 17 August 2016]

[7] L. Carla, R. Fantacci, F. Gei, D. Marabissi and L. Micciullo, "LTE Enhancement for Public Safety and Security Communications to support Group Multimedia Communications," IEEE Network, vol. 30, no. 1, pp. 80 - 85, 2016.

[8] TCCA, "Mobile Broadband for Critical Communications Users: A review of options for delivering Mission Critical solutions," TETRA and Critical Communication Broadband Group, White Paper, 2013.

[9] USA Government, "Middle Class Tax Relief and Job Creation Act of 2012," Public Law 112-96, 2012.

[10] NPSTC, "National Public Safety Telecommunications Council," [Online]. Available: http://www.npstc.org/firstNet. jsp. [Accessed August 2016].

[11] N. N. a. Solutions, "LTE-Advanced Evolution in Releases 12-14: New services to pave the way to 5G," Nokia Networks White Paper, 2015.

[12] R. Fantacci, F. Gei, D. Marabissi and L. Micciullo, "Public Safety Networks Evolution toward Broadband: Sharing Infrastructures and Spectrum with Commercial Systems," IEEE Communications Magazine, vol. 54, no. 4, pp. 24 - 30, 2016.

[13] A. Mason, "Public Safety Mobile Broadband and Spectrum needs," Final Report, 2010.

[14] R. Liebhart, D. Chandramouli, C. Wong and J. Merkel, LTE for Public Safety, Wiley, 2015.

[15] H. H. Hoang, R. Malhame and G. Chen, "Traffic Engineering of Trunked Land Mobile Radio Dispatched Systems," in IEEE Vehicular Technology Conference, 1991. Gateway to the Future Technology in Motion, St. Louis, MO, 1991.

[16] H. H. Hoang, R. Malhame and C. Rosenberg, "
Communication Load and Delay in Multichannel Land Mobile Systems for Dispatch Traffic: a Queuing model Analysis," in Vehicular Technology Conference, 1992, IEEE 42nd, Denver, CO, 1992.

[Online]. Available:

[17] N. Cackov, B. Vujicic, S. Vujicic and L. Trajkovic, "Using Network Activity Data to model the utilization of a Trunked Radio System," in Proc. SPECTS, San Jose, CA, 2004.

[18] N. Cackov, J. Song, B. Vujicic, S. Vujicic and L. Trajkovic, "Simulation and Performance Evaluation of a Public Safety Wireless Network: Case Study," SIMULATION, vol. 81, no. 8 , pp. $571-585,2005$.

[19] F. C. C. P. S. a. H. S. B. C. System, "Emergency Communications during the Minneapolis Bridge Disaster," Analysis Division, Technical case study, Minneapolis, 2008.

[20] M. Lin, W. Shouming and Q. Wei, "A Novel Traffic Analysis Method for PoC over LTE Based on Retrial Calling Model," in IEEE 2011 Communications and Networking in China (CHINACOM), 2011 6th International ICST Conference, Harbin, 2011.

[21] A. Ali, M. Alshamrani, A. Kuwadekar and K. Al-Begain, "Evaluating SIP Signaling Performance for VoIP over LTE Based Mission-Critical Communication Systems," in IEEE NGMAST, Cambridge, 2015.

[22] F. Gutierrez, A. Valdovinos and P. Perez, "Development of a traffic simulator for mobile communication networks based on TETRA technology," in Personal, Indoor and Mobile Radio Communications, 2002. The 13th IEEE International Symposium, 2002.

[23] 3GPP, "Proximity - Based Services (ProSe)," 3GPP TS 23.303.

[24] 3GPP, "Group Communication System Enablers for LTE (GCSE_LTE)," 3GPP TS 23.468.

[25] 3GPP, "Mission Critical Push to Talk MCPTT (Release 13)," 3GPP TS 22.179.

[26] ETSI, "Terrestrial Trunked Radio (TETRA); Voice plus Data (V+D); Designers' Guide; Part 2: Radio channels, network protocols and service performance," ETR 300 - 2, 1997.

[27] A. W. Graham, N. C. Kirkman and P. M. Paul, Mobile Radio Network Design in the VHF and UHF Bands: A Practical Approach, NJ: John Wiley \& Sons Inc.,, 2007.

[28] C. Cox, An Introduction to LTE, Wiley, 2014.

[29] A. ElNashar, M. A. El-saidny, and M. Sheriff, Design, deployment and performance of 4G- LTE networks: A Practical Approach, Wiley, 2014. 